\title{
Magnetoelastic Villari Effect in Structural Steel Magnetized in the Rayleigh Region
}

\author{
M. Kachniarz ${ }^{a, *}$, K. Kolakowska ${ }^{a}$, J. Salach ${ }^{a}$, R. SzewczyK ${ }^{a}$ And P. NowaK ${ }^{b}$ \\ ${ }^{a}$ Institute of Metrology and Biomedical Engineering, Warsaw University of Technology, \\ św. Andrzeja Boboli 8, 02-525 Warsaw, Poland \\ ${ }^{b}$ Industrial Research Institute for Automation and Measurements PIAP, \\ Al. Jerozolimskie 202, 02-486 Warsaw, Poland
}

\begin{abstract}
The following paper presents the results of investigation on the magnetoelastic Villari effect in structural steel magnetized in the so-called Rayleigh region, which corresponds to relatively low magnetizing fields, much lower than coercive field. Two grades of structural steel were investigated during the performed measurements. Obtained results indicate significant correlation between applied mechanical stress and magnetic properties of the investigated materials.
\end{abstract}

DOI: 10.12693/APhysPolA.133.660

PACS/topics: 75.30.-m, 75.60.-d, 75.50.Cc, 75.80.+q, 07.10.Lw

\section{Introduction}

One of the several magnetomechanical effects, besides magnetostriction or Wiedemann and Matteucci effects, is magnetoelastic Villari effect discovered by Emilio Villari in 1865 [1-3].

Magnetoelastic Villari effect is physical phenomenon involving change of magnetic state and properties of the magnetized ferromagnetic material under the influence of mechanical stress applied to the material [3, 4]. When the sample of the material is subjected to the mechanical stress, changes of magnetic flux density within the material and relative magnetic permeability of the material can be observed. This phenomenon could be utilized in magnetomechanical sensors of force and stress [5] or for non-destructive method of stress assessment in constructions made of ferromagnetic materials, like many grades of structural steel [6].

Most of the recent studies on application of Villari effect in stress assessment were performed with high magnetizing fields, corresponding to the saturation region of hysteresis loop of the material [7]. However, this method seems to be relatively hard for implementation, as it requires high number of windings in magnetizing coil and high magnetizing current. Therefore, it is very interesting to study the behavior of structural steel in low magnetizing fields, corresponding to the so-called Rayleigh region, to verify if there is a possibility to observe similar effect for such low fields, as it is in the saturation region.

\section{Rayleigh region}

The Rayleigh region was described for the first time by Lord John Rayleigh in 1887 [8]. It is a first part of the

*corresponding author; e-mail: m.kachniarz@mchtr.pw.edu.pl initial magnetization curve of the ferromagnetic material, where the dependence between magnetic flux density or magnetization of the material and magnetizing field (B-H or $\mathrm{M}-\mathrm{H}$ curve) can be described with the second degree polynomial equation known as Rayleigh law of magnetization [8]:

$$
B(H)=\mu_{0} \mu_{i} H+\mu_{0} \alpha_{R} H^{2},
$$

where $B$ is magnetic flux density, $H$ is magnetizing field and $\mu_{0}$ is magnetic permeability of free space. In the Rayleigh law there are two coefficients characterizing the ferromagnetic properties of the material: initial relative magnetic permeability $\mu_{i}$ and the so-called Rayleigh coefficient $\alpha_{R}$.

Rayleigh region is associated with the initial stage of magnetization process, where the main magnetization mechanism is movement of the magnetic domain walls and there is no rotation of the magnetic vectors of the domains. Initial magnetic permeability $\mu_{i}$ characterizes linear reversible changes of magnetic flux density $B$ under the influence of changing magnetizing field $H$, which are connected with elastic deflections of the domain walls within the material. Rayleigh coefficient $\alpha_{R}$ is describing nonlinear irreversible changes of $B$, which are the source of magnetic hysteresis phenomenon and originates from permanent translations of the domain walls.

It is not possible to determine general range of Rayleigh region in ferromagnetic materials as it is strongly dependent on the material. Previous researches indicate that for steel the range of Rayleigh region is about $0.5 \div 0.6$ of coercive filed $H_{c}$ measured in saturation region [9].

It may be expected that material coefficients will be affected by the mechanical stress applied to the sample of the ferromagnetic material. Therefore they may be utilized for stress assessment in constructional elements made of ferromagnetic grades of steel. 


\section{Investigated materials}

The subject of the investigation were two grades of structural steel exhibiting ferromagnetic properties. Both grades are widely utilized in the construction industry.

The first one was $\mathrm{C} 45$ medium carbon steel (carbon content $0.42 \div 0.50 \%$ ) of higher quality and strength. It is utilized for mechanical components like crankshafts, axes, gearwheels and medium-loaded pump rotors. It is mechanically characterized by yield strength over $350 \mathrm{MPa}$ and tensile strength about $600 \div 800 \mathrm{MPa}$.

The second investigated grade was $13 \mathrm{CrMo} 4-5$ heat resistant alloy steel with addition of chromium (about $0.70 \div 1.15 \%$ ) and molybdenum (about $0.40 \div 0.60 \%$ ). It has relatively low carbon content about $0.08 \div 0.18 \%$. It is mostly utilized in energy industry for manufacturing hot steam pipe lines, heating tubes and steam collectors as well as pressure vessels and turbine blades. Its yield strength is over $280 \mathrm{MPa}$ and its tensile stress is about $450 \div 600 \mathrm{MPa}$.

Both investigated materials were formed into frameshaped samples cut from the steel sheet of thickness 2 $\mathrm{mm}$. Each sample had two symmetrical columns, on which magnetizing (40 turns) and sensing (100 turns) windings were made.

\section{Measurement setup}

For performing the experiment, special measurement setup was utilized. The setup, presented in Fig. 1, was composed of digitally controlled system for investigation of magnetic characteristics of ferromagnetic materials and equipment for mechanical stress generation.

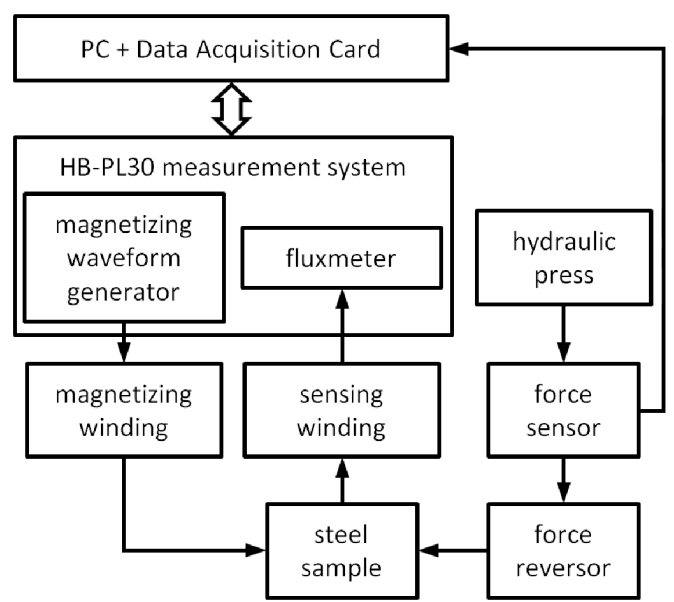

Fig. 1. Schematic block diagram of the measurement setup.

Measurement of magnetic characteristics of investigated materials was performed with HB-PL30 measurement system for investigation of ferromagnetic materials developed at Warsaw University of Technology. The system is composed of magnetizing waveform generator, which produces triangle or sinusoidal current waveform driving magnetizing winding, and fluxmeter, which measures magnetic flux density within the material on the basis of voltage induced in the sensing winding. The HB-PL30 is digitally controlled by the PC with Data Acquisition Card installed.

The mechanical stress applied to the investigated sample is generated with oil hydraulic press. Force generated by the press is measured with strain gauge force sensor. and the results are transmitted to the PC. In order to obtain tensile stress acting upon the sample, compressive force produced by the press is converted to the tensile force with special force reversor.

\section{Experimental results}

Each of investigated samples was subjected to the tensile stress from $0 \mathrm{MPa}$ to the rupture point. For each value of applied stress the family of Rayleigh hysteresis loops were measured with the magnetizing field amplitude $H_{m}$ form $50 \mathrm{~A} / \mathrm{m}$ to $400 \mathrm{~A} / \mathrm{m}$ and step $25 \mathrm{~A} / \mathrm{m}$.

On the basis of measurement results, the dependence of maximum magnetic flux density $B_{m}$ on the applied tensile stress $\sigma_{T}$ was determined for each material, which is presented in Fig. 2 and Fig. 3.

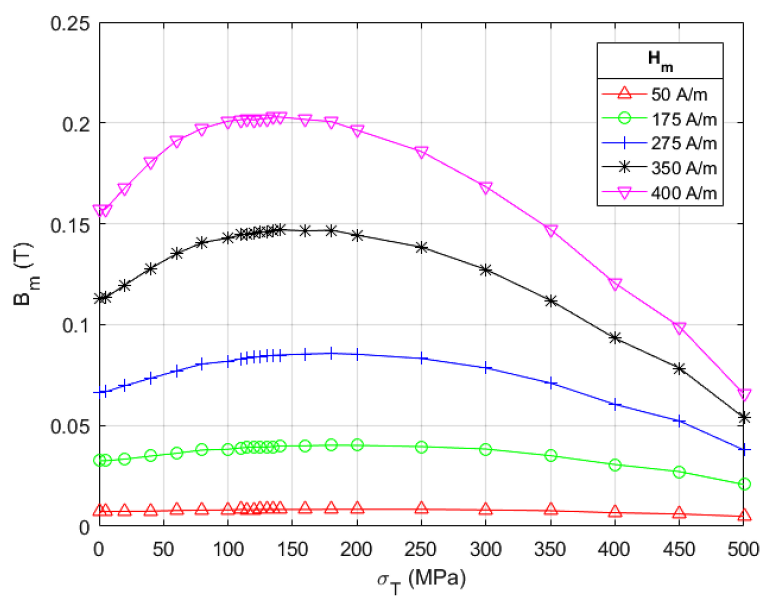

Fig. 2. Dependence of maximum magnetic flux density $B_{m}$ on the tensile stress $\sigma_{T}$ for C45 steel at selected values of magnetizing field amplitude $H_{m}$.

As it can be seen, for both investigated grades of steel obtained characteristics are similar. For low values of the tensile stress $\sigma_{T}$ the value $B_{m}$ is increasing up to the maximum value and then starts to decrease. The stress value corresponding to the maximum of $B_{m}$ is known as Villari reversal point [3] (140 MPa for C45 steel and $120 \mathrm{MPa}$ for 13CrMo4-5 steel). The observed phenomenon becomes stronger with increasing value of magnetizing field amplitude $H_{m}$.

For each value of applied tensile stress, the material coefficients: initial magnetic permeability $\mu_{i}$ and the Rayleigh coefficient $\alpha_{R}$ were determined on the basis of commutation curve obtained from vertices of the 


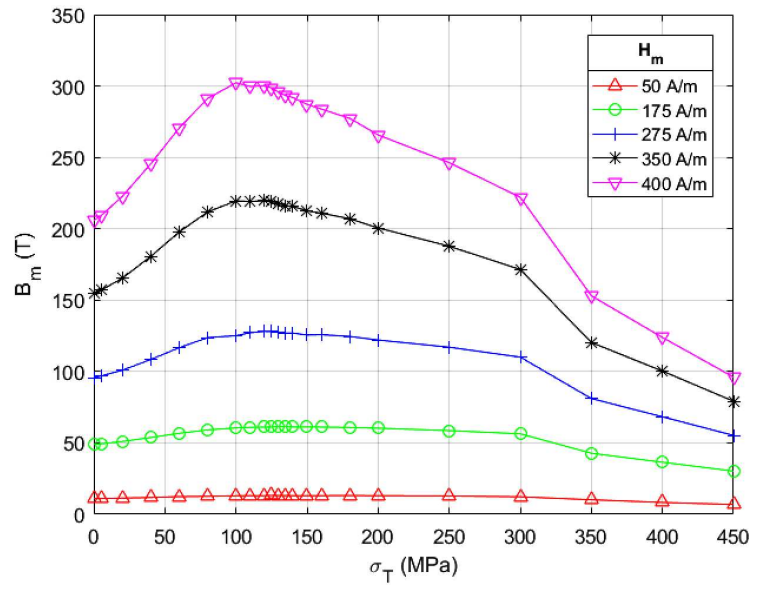

Fig. 3. Dependence of maximum magnetic flux density $B_{m}$ on the tensile stress $\sigma_{T}$ for $13 \mathrm{CrMo} 4-5$ steel at selected values of magnetizing field amplitude $H_{m}$.

Rayleigh hysteresis loops measured for increasing amplitude of magnetizing field $H_{m}$. The dependences of the material coefficients on the value of applied tensile stress $\sigma_{T}$ for each investigated steel are presented in Fig. 4 and Fig. 5 .

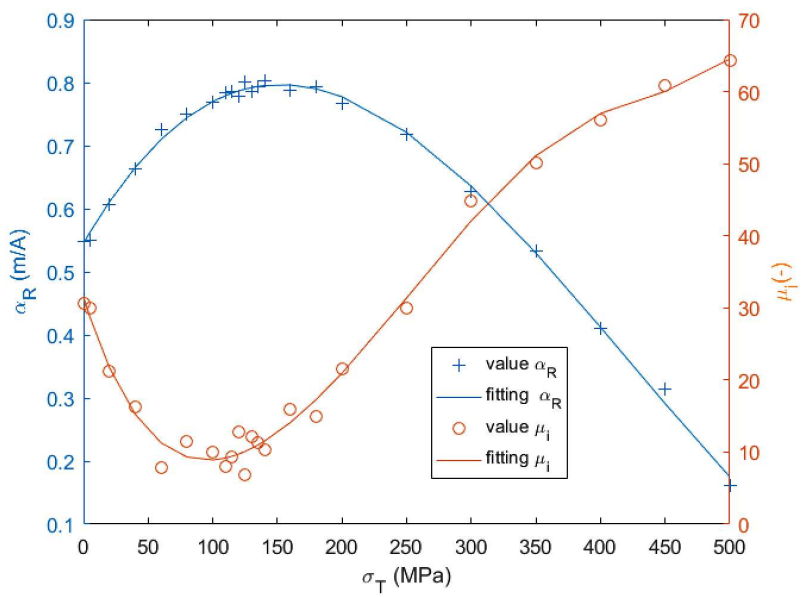

Fig. 4. Dependence of Rayleigh coefficient $\alpha_{R}$ and initial magnetic permeability $\mu_{i}$ on $\sigma_{T}$ for C45 steel (lines are polynomial approximations of the characteristics).

Both Rayleigh coefficient $\alpha_{R}$ and initial magnetic permeability $\mu_{i}$ are significantly changing with the value of tensile stress applied to the sample. Also both parameters are reaching their extrema for stress value equal the value of Villari point ( $\alpha_{R}$ reaches maximum, while $\mu_{i}$ reaches minimum). For both investigated grades of steel obtained characteristics exhibit very similar shape.

\section{Conclusions}

The paper presents the original results of investigation on the magnetoelastic properties of the structural steel in the Rayleigh region. The results obtained during the investigation indicate strong correlation between

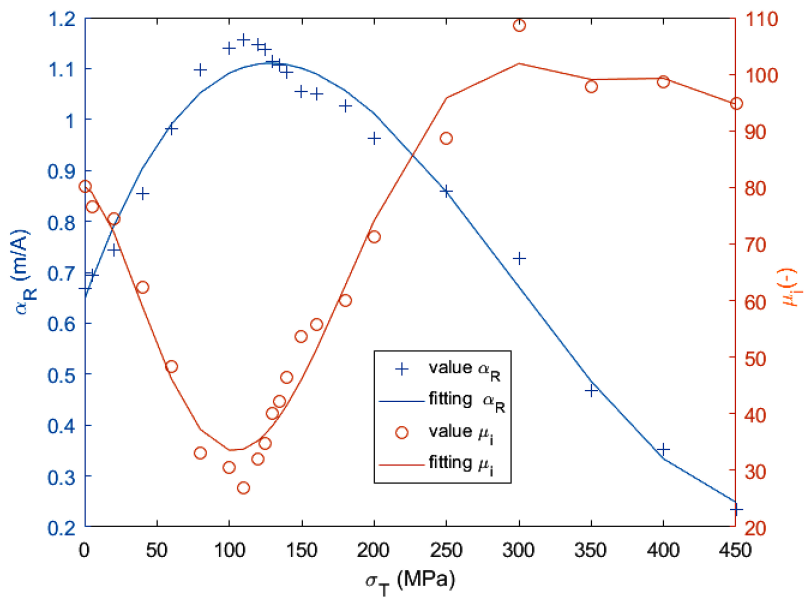

Fig. 5. Dependence of Rayleigh coefficient $\alpha_{R}$ and initial magnetic permeability $\mu_{i}$ on $\sigma_{T}$ for $13 \mathrm{CrMo} 4-5$ steel (lines are polynomial approximations of the characteristics).

mechanical stress applied to the structural steel and its magnetic properties in Rayleigh region.

For the first time the stress dependence of material coefficients in the Rayleigh region (Rayleigh coefficient and initial magnetic permeability) were determined for two grades of the structural steel. Obtained results are very similar for both investigated grades. This allows to suppose that there is a possibility of generalization of the obtained dependencies and development of the non-destructive methodology of stress assessment in ferromagnetic structural steel.

\section{Acknowledgments}

This work was partially supported by statutory founds of Institute of Metrology and Biomedical Engineering, Warsaw University of Technology (Poland).

\section{References}

[1] E. Hristoforou, A. Ktena, J. Magn. Magn. Mater. 316, 372 (2007).

[2] T. Charubin, A. Juś, in: Advances in Intelligent Systems and Computing, vol. 550, Eds. R. Szewczyk, C. Zieliński, M. Kaliczyńska, Springer, Berlin 2017, p. 527.

[3] A. Bieńkowski, J. Magn. Magn. Mater. 215-216, 231 (2000).

[4] H.B. Wang, Z.H. Feng, IEEE Trans. Magn. 49, 1327 (2013).

[5] Y.Y. Kim, S.H. Cho, H.C. Lee, J. Sound Vib. 268, 799 (2003).

[6] E.S. Gorkunov, A.M. Povoltskaya, K.E. Solov'ev, S.M. Zadvorkin, Russian Journal of Nondestructive Testing 46, 638 (2010).

[7] S. Bao, S.F. Gong, J. Appl. Phys. 112, 113902 (2012).

[8] Lord Rayleigh, Philos. Mag. 23, 225 (1887).

[9] M. Kachniarz, R. Szewczyk, Acta Phys. Pol. A 131, 1244 (2017). 